\title{
Sulbactam Pivoxil
}

National Cancer Institute

\section{Source}

National Cancer Institute. Sulbactam Pivoxil. NCI Thesaurus. Code C96733.

The pivoxil prodrug form of sulbactam, a semi-synthetic beta-lactamase inhibitor, with beta-lactam antibiotic adjuvant activity. After oral administration of sulbactam pivoxil, the ester bond is cleaved, releasing active sulbactam. The beta-lactam ring of sulbactam irreversibly binds to beta-lactamase at or near its active site, thereby blocking enzyme activity and preventing metabolism of other beta-lactam antibiotics by the enzyme. 